\title{
Creencias sobre el amor romántico y las relaciones íntimas: implicaciones en jóvenes de la Comunidad de Madrid¹5
}

\author{
Enrique Bonilla-Algovia \\ Ms. en Psicopedagogía \\ Universidad de Alcalá, España \\ Correo electrónico: enrique.bonilla@uah.es
}

Esther Rivas-Rivero

PhD. en Ciencias de la Educación

Universidad de Alcalá, España

Correo electrónico: esther.rivas@uah.es
Recibido: 02/03/2020

Evaluado: 01/04/2020

Aceptado: 27/04/2020

\section{Resumen}

El objetivo de este estudio ha sido conocer las creencias sobre el amor y sobre las relaciones íntimas que tiene un grupo jóvenes de la Comunidad de Madrid, así como explorar la posible relación entre estas creencias y la desigualdad de género en el ámbito de la pareja. La investigación se ha basado en un enfoque metodológico cualitativo y la información se ha recogido mediante la técnica de grupos focales. Participaron 34 estudiantes de la Universidad de Alcalá, con edades comprendidas entre los 18 y los 34 años ( $M=21.32$; DT = 3.12). Los resultados muestran que las creencias que tienen los hombres y las mujeres son distintas y parecen estar relacionadas con los mandatos de género tradicionales. La visibilización del imaginario sobre el amor en la juventud puede favorecer la puesta en marcha de programas preventivos que confronten las creencias desigualitarias y fomenten modelos relacionales basados en la igualdad, el respeto y la no violencia.

\section{Palabras clave}

Amor, amor romántico, desigualdad de género, relaciones de pareja.

15 Para citar este artículo: Bonilla-Algovia, E. y Rivas-Rivero, E. (2021). Creencias sobre el amor romántico y las relaciones íntimas: implicaciones en jóvenes de la Comunidad de Madrid. Informes Psicológicos, 21(2), pp. 243-257 http://dx.doi.org/10.18566/infpsic.v21n2a15.

Este trabajo se ha realizado con el apoyo de un Contrato Predoctoral de Formación de Profesorado Universitario de la Universidad de Alcalá, España. 


\title{
Beliefs about romantic love and intimate relationships: implications for young people in the Community of Madrid
}

\begin{abstract}
This study aimed to find out the beliefs about love and intimate relationships held by a group of young people from the Community of Madrid. It also explored the possible relationship between these beliefs and gender inequality in the heterosexual couple. The research was based on a qualitative methodological approach and the information was collected using the focus group technique. 34 students from the University of Alcalá participated, aged between 18 and 34 years $(M=21.32 ; S D=$ 3.12). The results show that men's and women's beliefs are different and seem to be related to traditional gender mandates. The visibility of the imaginary about love in youth can favor the implementation of preventive programs capable of confronting unequal beliefs and promoting relational models based on equality, respect and non-violence.
\end{abstract}

\section{Keywords} Love, romantic love, gender inequality, couple relationships.

\section{Crenças sobre o amor romântico e as relações íntimas: implicações para os jovens da Comunidade de Madrid}

\section{Resumo}

0 objetivo deste estudo foi conhecer as crenças sobre 0 amor e as relações íntimas de um grupo de jovens da Comunidade de Madrid, explorando as possíveis relações entre essas crenças e a desigualdade de gênero no campo do casal heterossexual. A pesquisa tem uma abordagem metodológica qualitativa e as informações foram coletadas por meio da técnica de grupo focal. Participaram 34 alunos da Universidade de Alcalá, com idades compreendidas entre os 18 e os 34 anos $(M=21.32 ; \mathrm{DT}=3.12)$. Os resultados mostram que as crenças de homens e mulheres são diferentes e parecem estar relacionadas aos mandatos tradicionais de gênero. A visibilidade do imaginário sobre 0 amor na juventude pode favorecer a implementação de programas preventivos que enfrentem crenças desiguais e promovam modelos relacionais baseados na igualdade, respeito e não violência. 


\section{ntroducción}

Los seres humanos, a lo largo de la historia, han tenido especial interés en el amor y en las relaciones íntimas; sin embargo, no es hasta la segunda mitad del siglo XX cuando el amor recibe la atención de la comunidad científica. En la década de los 70 se convierte en objeto de estudio de diferentes disciplinas (Cassepp-Borges \& Leôni, 2009), entre las que destacan la sociología, la historia, la psicología y los estudios feministas (García \& Cedillo, 2011). En este sentido, el abordaje científico del amor es complejo porque da cabida a distintos enfoques teóricos y perspectivas metodológicas (Bosch, Ferrer, Ferreiro \& Navarro, 2013; Ferreiro, Caro, Ferrer, Casado \& Casado, 2018).

Partiendo de una perspectiva culturalista, se entiende el amor como una construcción social que varía en función del momento histórico y de la cultura predominante (Barrón, Martínez-Íñigo, De Paúl \& Yela, 1999; Bonilla \& Rivas, 2018; Lagarde, 2001). En el ámbito de las relaciones íntimas, el fenómeno amoroso puede entenderse como la combinación de las emociones, las cogniciones y los comportamientos que forman parte de la relación e interaccionan entre sí (Baron \& Byrne, 2005). La forma en la que estos elementos (emociones, cogniciones y comportamientos) se interrelacionan, se expresan y se experimentan en las relaciones íntimas, tiene su explicación en los procesos de socialización que se dan en los distintos contextos sociohistóricos. A través de la socialización recibida en los diferentes espacios (familia, sistema educativo, medios de comunicación, medios de divulgación cultural, etc.), se configuran las creencias sobre el amor, se interiorizan los mitos románticos y se conforma el imaginario sobre el funcionamiento de las relaciones íntimas (Ferrer \& Bosch, 2013). Por lo tanto, de acuerdo con la literatura en la temática, el amor está sujeto al aprendizaje cultural y depende en gran medida de los procesos de socialización (Bosch et al., 2013; García et al., 2011; Pascual, 2016).

La socialización que reciben mujeres y hombres en las sociedades patriarcales es distinta y está articulada en torno a la construcción social del género (Colás, 2007). Esto es lo que se llama socialización diferencial y consiste en la transmisión de roles, prescripciones, normas y mensajes diferenciados según sean dirigidos a hombres o mujeres (Espinar, 2007; Ferrer \& Bosch, 2013). La masculinidad, desde la cultura patriarcal, se vincula con cualidades como la independencia, la dominación, la fuerza, la no emocionalidad, etc. La feminidad, en cambio, se vincula con cualidades como la dependencia, la pasividad, los cuidados, la emocionalidad, etc. Todos estos mandatos y roles, en tanto se atribuyen de forma diferenciada y tienen una valoración social desigual, configuran un sistema relacional jerárquico (Bosch, Ferrer \& Alzamora, 2005) y, a su vez, influyen en la vivencia del amor y en el funcionamiento de las relaciones íntimas (Ferreiro et al., 2018). La socialización diferencial es, en consecuencia, una estrategia de la estructura patriarcal para perpetuar y naturalizar la división sexual del trabajo y las desigualdades sociales entre hombres y mujeres (Ferrer \& Bosch, 2013).

La desigualdad de género, desde la perspectiva feminista, es una 
problemática estructural y multidimensional que se produce y reproduce en ámbitos muy variados de las esferas pública y privada (Alfama, Cruells \& De La Fuente, 2014): "a nivel micro, meso y macrosocial, desde lo subjetivo, las relaciones interpersonales, las estructuras sociales, laborales, económicas, políticas y normativas, y que cuenta con una dimensión material pero también se enraíza fuertemente en estereotipos sociales, valores y subjetividades" (p. 214). En este sentido, uno de los ámbitos en los que la desigualdad de género tiene lugar son las relaciones íntimas heterosexuales. Este hecho ha supuesto que uno de los principales objetivos de los Estudios de Género y Feministas en la actualidad sea analizar el papel que las creencias sobre el amor tienen en la reproducción de la desigualdad en el seno de la pareja (Verdú, 2014). Estudiar los vínculos sentimentales y las creencias al respecto es fundamental para explicar los mecanismos de subordinación de las mujeres y la asimetría en las relaciones íntimas (Esteban, 2009; Rodríguez-Santero, García-Carpintero \& Porcel, 2017). Por lo tanto, el objetivo del presente trabajo ha sido conocer las creencias sobre el amor y sobre las relaciones íntimas que tiene un grupo de jóvenes de la Comunidad de Madrid y explorar su posible relación con la desigualdad de género en el ámbito de la pareja heterosexual.

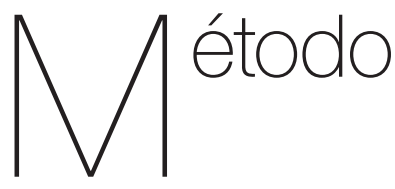

Participantes

La muestra del estudio está compuesta por 34 estudiantes de la
Universidad de Alcalá, 9 hombres y 25 mujeres, que se encontraban cursando estudios de grado en la Facultad de Educación, concretamente estudiaban Magisterio en Educación Primaria. La edad media de la muestra fue 21.32 años (DT = 3.12) y el rango de edad, 18 a 34 años. La selección de las personas que participaron en el estudio se realizó mediante un muestreo de conveniencia e intencional. Así, entre el alumnado de la Universidad de Alcalá, se establecieron los siguientes criterios de inclusión muestral: ser mayor de edad (1), tener o haber tenido al menos una relación de pareja (2) y estudiar un grado en la Facultad de Educación (3). En el momento de la investigación, el 41.2\% tenía pareja y el 58.8\% no la tenía. El hecho de que en el estudio participasen más mujeres que hombres tiene una explicación contextual y se debe a la feminización de la docencia en las primeras etapas educativas, como síntoma de la división sexual del trabajo y de las tareas y roles sociales (Sánchez, 2012).

\section{Instrumento}

La información fue recogida mediante la técnica denominada grupos focales (focus groups). Esta técnica se enmarca en la metodología cualitativa y permite acceder en profundidad a pensamientos, sentimientos y experiencias (Krueger, 1991). Las estrategias cualitativas de trabajo en grupo, al llevarse a cabo en un ambiente de interacción, facilitan la discusión y la fluidez en la comunicación (Hamui-Sutton \& Varela-Ruiz, 2013). Se trata de una especie de entrevistas grupales que tienen lugar en un ambiente relajado y en las que se conversa sobre diferentes temas de manera 
informal, siempre bajo la conducción de una persona experta en la materia (Hernández, Fernández \& Baptista, 2010), por lo que las respuestas que van surgiendo son el material de estudio (Bohórquez \& Rodríguez-Cárdenas, 2014). El análisis del material se realizó desde la Teoría Fundamentada (Glaser \& Strauss, 1999). Así, se utilizó un guion fluido de preguntas para generar teoría a partir de la interpretación del fenómeno del amor en las personas participantes. Los temas del guion fueron el significado del amor, la vivencia de las relaciones íntimas, las experiencias amorosas previas y los mitos del amor romántico. Finalmente, se realizaron cuatro grupos focales no mixtos: primer grupo, 9 hombres; segundo grupo, 9 mujeres; tercer grupo, 8 mujeres; y cuarto grupo, 8 mujeres. Esta distribución se realizó con el fin de reducir la deseabilidad social y las presiones ante la presencia de personas del sexo opuesto.

\section{Procedimiento}

El profesorado de la Universidad de Alcalá informó al alumnado de la Facultad de Educación sobre la investigación que se iba a realizar. La información facilitada giró en torno a los objetivos y las características del estudio. De todas las personas que quisieron participar, fueron seleccionadas las 34 que cumplían con los criterios de inclusión muestral. Antes de iniciar los grupos focales, se explicó el procedimiento de la investigación y se facilitó un cuestionario anónimo en el que se recogían los datos sociodemográficos. La participación fue voluntaria y contó con el consentimiento informado. Las sesiones se desarrollaron en las instalaciones de la Universidad de Alcalá y se grabaron mediante dispositivos de recogida de audio. La duración de los grupos focales osciló entre los 70 y los 120 minutos.

\section{Análisis}

La información en formato audio se clasificó mediante la asignación de uno o varios códigos, creando así un sistema de categorías. Esto permitió un acceso rápido a las mismas y facilitó el análisis del contenido. Los datos se organizaron en categorías en función de sus diferencias y similitudes entre sí (Hernández et al., 2010). Las categorías emergieron de los datos. Las tres que se contemplaron en el presente trabajo son: "cualidades que se buscan en una pareja", "expectativas y objetivos de las relaciones íntimas" y "relación entre amor y felicidad". Para la presentación de los resultados, se transcribieron de forma literal los discursos más representativos. Se comparó la información codificada y se seleccionaron las citas que mejor representaban las categorías de análisis. El texto entre corchetes ha sido introducido por los investigadores para hacer aclaraciones; los puntos suspensivos se utilizan para indicar una pausa dubitativa o una prolongación de la última sílaba; y el paréntesis con puntos suspensivos (...) indica que una parte de la cita original ha sido eliminada. Los datos se procesaron y analizaron de forma anónima, asignando un código a cada cita, que representa el grupo focal de referencia (G1, G2, G3 o G4), el sexo (hombre o mujer) y el número asignado a cada persona (1, 2, 3...). Cuando algún o alguna participante se dirigía a una persona en concreto, se ha sustituido el nombre por el código que le representa. 


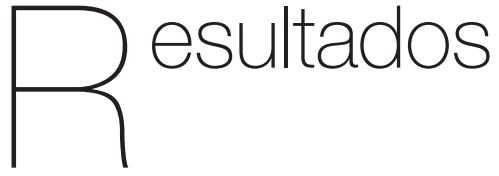

Los resultados del presente trabajo, en línea con otras investigaciones que han abordado las creencias sobre el amor y/o sobre las relaciones íntimas en la población española (Barrón et al., 1999; Cerreti \& Nararro, 2018; García et al., 2011; Rodríguez, Lameiras \& Carrera, 2015; Rodríguez-Santero et al., 2017), muestran que las visiones de las mujeres y de los hombres son distintas. No obstante, la diferencia entre este y otros estudios radica en su metodología, ya que la utilización de técnicas cualitativas permite profundizar en las creencias sobre el amor y facilita la comprensión de la influencia que estas creencias pueden tener en la configuración y el funcionamiento de las relaciones íntimas (Cubells \& Calsamiglia, 2015; Esteban, 2009; Esteban \& Távora, 2008; Ferreiro et al., 2018). A continuación, se analizan los datos cualitativos atendiendo a las tres categorías establecidas.

\section{Categoría 1. Cualidades que se buscan en una pareja}

Los mandatos de género que se interiorizan mediante la socialización diferencial parecen condicionar las cualidades que los hombres y las mujeres buscan en una pareja (Bosch et al., 2013). En primer lugar, analizando las respuestas de los hombres, se encuentran dos posiciones diferenciadas. La primera es la de quienes no buscan unas cualidades concretas en la otra persona. La segunda, en cambio, es la de quienes buscan características como cuidados y atención emocional. En este sentido, como se muestra en los siguientes comentarios, una parte de los hombres busca una relación en la que la mujer se responsabilice del sostenimiento de los vínculos amorosos y de su bienestar individual, es decir, una mujer que tenga las cualidades que caracterizan al rol femenino tradicional. Chaves (2012) expone que los papeles asociados a la masculinidad y la feminidad suponen, por un lado, que los hombres primen su individualidad y su éxito en la esfera pública y, por otro lado, que las mujeres interioricen como propias las necesidades ajenas. En esta misma línea, los participantes de la segunda posición se liberarían de los cuidados y del sostenimiento de las relaciones íntimas atribuyendo y responsabilizando a las mujeres de estas tareas.

"Mi prototipo de mujer sería una mujer que por ejemplo a mí me tenga con los pies en el suelo, porque yo soy muy alocado, a mí me gusta hacer muchas locuras, y yo por ejemplo necesito una persona a mi lado que me diga, cálmate, piensa las cosas y actúa en consecuencia a lo que haces. Una persona que a mí me llegue, me coja y, por ejemplo, si soy un desfasado por ejemplo saliendo de fiesta, que a mí me coja y me diga, controla. No una persona por ejemplo que esté muy encima de mí, sino que me deje una rienda suelta, pero que tampoco me deje aquí hacer todo lo que yo quiera. Y también una mujer que, por ejemplo, sobre todo me entienda, que yo creo que eso es muy difícil porque, como he dicho, mi cabeza es muy alocada". (G1, hombre 1) 
"[Lo que busco es una mujer] que me tenga también con los pies en el suelo, porque soy una persona que se me va mucho la olla. Entonces, que me diga, cálmate, piensa las cosas y vamos a hacer algo más". (G1, hombre 2)

Las sociedades patriarcales están configuradas de tal manera que el control estructural de los hombres requiere del trabajo reproductivo de las mujeres en la esfera privada (Expósito, Moya \& Glick, 1998). Esta dependencia diádica que los hombres tienen de las mujeres como madres y esposas ha suscitado una ideología sexista que tiene como objetivo mantener el poder estructural en manos de los hombres y perpetuar el trabajo reproductivo en manos de las mujeres (Glick \& Fiske, 1996). Esta ideología, oculta bajo sentimientos aparentemente positivos, idealiza a las mujeres que se adaptan a los roles y espacios tradicionalmente femeninos, promueve la complementariedad de los papeles diferenciados de género y ensalza a las mujeres por su rol de madres y esposas. En este sentido, la posición de los participantes que buscan cuidados y atención emocional estaría vinculada con la ideología sexista en su componente benévolo y se ejemplificaría a la perfección en el siguiente comentario, en el que uno de los hombres manifiesta que le gustaría tener como pareja a una mujer con cualidades similares a las de una madre. Esto podría conceptualizarse como "maternización del amor" y aludiría a la búsqueda en la pareja de las características estereotípicas femeninas en las que se ha socializado a las mujeres y que la maternidad tradicional suele representar: entrega, cuidados, atención, abnegación, renuncia, etc. En consecuencia, la dependencia diádica que los hombres tienen de las mujeres en las sociedades patriarcales puede tener como resultado que estos idealicen las características estereotípicas femeninas y conviertan en objetos de amor a las mujeres que las encarnan, fomentando la adhesión a las mismas.

\begin{abstract}
"La personalidad que me gusta a mí es una personalidad que sea fuerte, que... o sea, una mujer que sea fuerte y que, a la vez, como ha dicho [hombre 1], que sepa un poco ponernos... vamos, ponerme a mí en mi lugar. ¿Sabes? Que diga, oye, que esto es así y es así. ¿Sabes? Básicamente un poco como mi... como nuestra madre. [...] Parece una tontería, pero, al fin y al cabo, yo creo que siempre buscamos algo parecido a lo que hay". (G1, hombre 5)
\end{abstract}

Las mujeres, como se muestra a continuación, buscan en la pareja cualidades muy diferentes a las expuestas por los hombres. Existe una amplia coincidencia en que el respeto, la igualdad y la empatía deben ser la base sobre la que construir las relaciones íntimas. El análisis de los discursos de las mujeres muestra que la palabra respeto es la que más se menciona en la sección de cualidades que se buscan en una pareja. Esto sugiere que, a lo largo de su vida amorosa, han podido experimentar situaciones en las que no han sido respetadas e, incluso, situaciones en las que han sido maltratadas, como expresan algunas participantes. La igualdad es una cualidad que, como ya se ha encontrado en otras investigaciones (Verdú, 2014), tiene presencia en el discurso de las mujeres pero no en el de los hombres. La unanimidad del discurso de las mujeres respecto a la búsqueda 
de respeto e igualdad en las relaciones íntimas muestra el calado que están teniendo los postulados feministas en la actualidad; sin embargo, también muestra que las mujeres tienen que seguir exigiendo una igualdad que les corresponde en un sentido formal, pero que se les niega sistemáticamente en la vida real. Esta situación se ejemplifica en que los hombres reclaman la desigualdad concreta que constatan en la cotidianeidad y las mujeres reclaman la igualdad abstracta que las leyes le garantizan y no llegan a constatar (De Beauvoir, 2008). Por lo tanto, las diferencias entre los hombres y las mujeres en cuanto a las cualidades que se buscan en una pareja muestran que la desigualdad sigue reproduciéndose en las relaciones íntimas y desenmascaran el espejismo de la igualdad (García, 2006).

"Físicas, a mí eso me da igual. Pero en cuanto a forma de ser, sobre todo respeto. Una persona con la que se pueda hablar, obviamente, y también pues que sea divertida y que te lo pases bien con él. Y sobre todo eso, que respete mis gustos, al igual que yo respeto los suyos". (G2, mujer 8)

"[Mi prototipo es una persona] que te apoye en tus decisiones. O sea, si tú quieres hacer algo o... Ya no solo en los estudios, sino en cualquier decisión que tomes, le puede parecer mejor o peor, o si estuviese en tu caso lo haría o no, pero que lo respete y que te apoye". (G3, mujer 12)

"Yo de una relación espero respeto e igualdad, ante todo. O sea, lo único que espero es eso. Bueno, lo mínimo que espero". (G2, mujer 7)
"Para mí también que sea empático; que tenga la capacidad de ponerse en mi lugar me parece súper fundamental. Aparte del respeto, por supuesto, porque va a haber una convivencia y va a haber diferentes opiniones y van a ocurrir mil cosas en pareja, que, si tiene la capacidad de ponerse en tu lugar, creo que se lleva de mejor forma a que si solo se queda con lo que él piensa o él siente". (G3, mujer 10)

"Yo creo que sí, que al final es el respeto porque... partiendo del respeto, no te va a... A ver... No te va a ser infiel, te va a respetar en tus decisiones, no va a influir en ellas de forma consciente. (...) O sea, para mí el respeto es lo más importante, dejando a un lado ya lo físico. Y también es importante la personalidad, por supuesto. Pero ya es lo que cada uno busque y si lo encuentra, porque tampoco vas a decidir si la persona en la que te has fijado lo tiene o no. Te fijas en si te respeta o no. Si no, no te merece la pena estar con esa persona". (G3, mujer 15)

Las mujeres, si bien dan importancia al respeto y la igualdad en sus discursos, consideran que las dinámicas relacionales dificultan su materialización en la cotidianeidad. Asimismo, un subgrupo de mujeres manifiesta que el amor es un sentimiento irracional e incontrolable y que, como consecuencia, lo que acontece en el contexto de la relación íntima puede llegar a serlo. Es decir, consideran que, del mismo modo que el sentimiento amoroso es incontrolable, lo que ocurre en las interacciones amorosas también escapa a su control. Estas creencias 
implican una situación de ambivalencia en el plano de las relaciones íntimas, de la que se desprende que el amor y el sufrimiento pueden ser compatibles y pueden darse de forma simultánea. Tal y como se ha encontrado en otras investigaciones, la construcción del amor como un sentimiento mágico, irracional y fuera de control no solo genera las condiciones que posibilitan la asimetría en el ámbito de la pareja, sino que favorece que las mujeres permanezcan en la relación incluso si esta es perjudicial y/o violenta (Cubells \& Calsamiglia, 2015). Esto mismo puede apreciarse en el presente estudio, de manera que este tipo de creencias, que están vinculadas al amor romántico, dejarían a las mujeres en una situación de vulnerabilidad ante la desigualdad y ante la violencia de género.

"Es que yo creo que el amor elimina - paraliza nuestra capacidad racional en muchos casos. Es decir, nuestro yo racional, que en cualquier otra situación actuaría, en el amor a veces es como si estuviera desconectado. $\bigcirc$ sea, ¿no os ha pasado alguna vez de decir, pero qué cojones estoy haciendo? En plan, yo esto no lo haría normal. Porque te gusta alguien mucho, mucho, mucho. En plan, ay, voy a hacer esto. Es como, me estoy humillando, qué chorrada. A veces nuestro yo racional, el yo que nosotras tenemos, o sea, nuestra visión de nosotras mismas, como que de repente ya no está en ese momento en el que nos enamoramos". (G2, mujer 9)

"Yo creo que todo el mundo realmente nos ponemos unos límites, pero que luego igual también es eso, que el amor realmente te ciega. $Y$ también depende de muchas cosas. Depende de la dependencia que tengas con tu pareja. Si estás como súper enamorada, pues igual eso, dejas pasar ciertas cosas que dices, joe, es que como le quiero tanto..." (G2, mujer 3)

\section{Categoría 2. Expectativas y objetivos de las relaciones íntimas}

Las expectativas y los objetivos acerca de las relaciones íntimas pueden tener un efecto directo en los papeles que adquiere cada miembro de la relación. La Tabla 1 muestra los discursos más representativos de las mujeres y los hombres en esta categoría. La literatura académica reporta que la socialización diferencial ha situado el amor en el centro de la vida de las mujeres y, sin embargo, en un plano secundario de la vida de los hombres (Ferrer \& Bosch, 2013; Leal, 2007). Los resultados, en esta misma línea, muestran que la visión de los hombres se caracteriza por la ausencia de expectativas de futuro; tienen la mirada puesta en el disfrute de la relación en el presente y no se plantean objetivos a largo plazo. La mayoría de las mujeres, en cambio, entiende el amor como un proyecto de vida; buscan compartir la vida con la persona amada y tienen la mirada puesta en el mantenimiento de la relación y en el crecimiento conjunto. La centralidad del amor en la vida de las mujeres y no en la de los hombres puede generar una plusvalía emocional en beneficio de estos, debido a que las mujeres, en comparación con los hombres, dedicarán una mayor 
cantidad de esfuerzos y cuidados en las relaciones íntimas (Jonásdóttir, 1993, citado en Ferreiro et al., 2018). En otras palabras, una dedicación no recíproca a la relación y a la pareja supondría que los hombres extraigan una plusvalía de atención y cuidados de las interacciones con las mujeres.

Tabla 1.

Expectativas y objetivos de las relaciones intimas.

\begin{tabular}{|c|c|}
\hline Hombres & Mujeres \\
\hline $\begin{array}{l}\text { "Yo lo que creo que es importante tam- } \\
\text { bién es, sobre todo al principio, no es- } \\
\text { perar nada de la relación. O sea, disfru- } \\
\text { tarla sin esperar nada, sin hacerte más } \\
\text { ilusiones de adónde vamos a llegar, de } \\
\text { qué va a pasar..., sino saber qué es lo } \\
\text { que estás viviendo en cada momento y } \\
\text { saber que, si esa persona es importante } \\
\text { para ti, va a seguir a tu lado. Entonces, } \\
\text { no es pensar en qué me va a aportar de } \\
\text { aquí a dos o doce años, sino qué me va } \\
\text { a aportar de aquí a hoy". (G1, hombre } 5)\end{array}$ & $\begin{array}{l}\text { "Yo creo que el objetivo... Vamos, por lo menos el que yo me marco con mi pareja } \\
\text { es compartir mi vida. Con lo bueno, con lo malo, con lo regular, con lo que venga". } \\
\text { (G2, mujer 6) } \\
\text { "Yo creo que el objetivo... Vamos, por lo menos el que yo me marco con mi pareja } \\
\text { es compartir mi vida. Con lo bueno, con lo malo, con lo regular, con lo que venga". } \\
\text { (G2, mujer 6) } \\
\text { "El amor, en sí, es querer a una persona y quererla con todos sus defectos y sus } \\
\text { cualidades. Y que esa persona, no digo que se convierta en tu prioridad, pero se } \\
\text { convierte en algo muy importante de tu vida. Y sobre todo querer que esa persona, } \\
\text { querer que esa persona esté bien". (G3, mujer 16) }\end{array}$ \\
\hline $\begin{array}{l}\text { "Seguir como ahora. Es decir, yo me } \\
\text { aplico la filosofía que tú has dicho [se } \\
\text { refiere al hombre 5]. No me gusta hablar } \\
\text { de futuro porque yo no sé, ni a nivel de } \\
\text { pareja ni a nivel de nada, yo no sé (...) } \\
\text { qué va a ser de mí en cinco años o qué } \\
\text { va a ser de mí el año que viene. Yo soy } \\
\text { feliz ahora y estoy a gusto ahora. } 0 \text { sea, } \\
\text { no espero nada, simplemente disfruto lo } \\
\text { que tengo ahora". (G1, hombre 6) }\end{array}$ & $\begin{array}{l}\text { "Y también eso, un poco con lo de futuro, que, a ver, tampoco que tengamos las } \\
\text { mismas al cien por cien expectativas, pero que, a lo major, crecer juntos es algo } \\
\text { importante también. (...) Como que también quiera crecer y evolucionar conmigo en } \\
\text { este caso, con la pareja". (G3, mujer 14) } \\
\text { "Yo si estoy con una pareja... Por ejemplo, yo con mi novio llevo mucho tiempo, yo } \\
\text { me planteo tener un futuro e irme a vivir fuera de mi casa y, a lo mejor, bueno, tener } \\
\text { hijos o no. Pero yo quiero cosas en mi vida a la larga que, si no las comparto con } \\
\text { esa persona, sé que no voy a llegar a eso. Entonces tengo que decidir si me gusta, } \\
\text { o sea, si quiero compartir con esa persona (...) aun teniendo esos principios que él } \\
\text { tenga o no, para tenerlo en mi vida a la larga". (G2, mujer 5) }\end{array}$ \\
\hline
\end{tabular}

\section{Categoría 3. Relación entre amor y felicidad.}

El modelo de amor romántico de la cultura occidental está compuesto por una serie de mitos que representan las creencias de la sociedad en torno a la naturaleza del amor (Barrón et al., 1999). Uno de estos mitos románticos se basa en la creencia de que las personas no pueden ser felices sin una relación de pareja; sin embargo, aunque este tipo de creencias parezcan neutras, los hombres y las mujeres las interiorizan de manera distinta debido a la diferenciación de roles sociales que se da en las sociedades patriarcales (Bonilla \& Rivas, 2018). En este sentido, los discursos de los hombres que participaron en el estudio muestran que, aunque valoran la importancia del amor en sus vidas, consideran que su individualidad está por encima de las relaciones íntimas y que su felicidad no depende de la tenencia de pareja. Así, en línea con la teoría de la socialización diferencial y los roles asociados a la masculinidad (Bosch et al., 2013; Ferreiro et al., 2018), los resultados parecen indicar que los hombres son socializados para que su bienestar y su gratificación provengan de 
la esfera pública y no del amor de pareja, de forma opuesta a lo que ocurre con las mujeres.

"Por encima de tu pareja estás tú". (G1, hombre 8)

"El sentimiento que tienes que tener hacia ti mismo, obviamente, pues... pienso que tiene que ser fuerte. (...) O sea, yo, por ejemplo, cuando no estoy en pareja, no me siento incompleto, me siento yo". (G1, hombre 1)

La centralidad del amor en la vida de las mujeres puede conllevar que el amor se viva como una necesidad y no como una opción. En esta línea, tal y como se muestra en la Tabla 2, las entrevistas muestran que, para una parte de las mujeres participantes, tener una relación íntima es un requisito indispensable para alcanzar la felicidad, es decir, supeditan su felicidad a la tenencia de pareja. Estos discursos pueden deberse a que el patriarcado ha configurado la creencia de que es el amor lo que da sentido a la vida de las mujeres (De Beauvoir, 2008; Herrera, 2014; Lagarde, 2001) y, en consecuencia, pueden desembocar en subordinación y adaptación de las mujeres al rol de género femenino con el fin de mantener el vínculo y la relación íntima (Esteban \& Távora, 2008). Hay otra parte de las mujeres, en cambio, que considera que su felicidad no depende de la tenencia de una relación íntima y que el amor de pareja no es lo que da sentido a sus vidas. Este tipo de creencias no solo se oponen al amor patriarcal, sino que, además, plantean una alternativa. El hecho de que las mujeres interpreten el amor como una elección y no como una necesidad facilita su emancipación individual y social, reduce las posibilidades de depender emocionalmente de la pareja y permite cuestionar la maternización del amor y la plusvalía emocional que caracteriza a las relaciones de poder históricamente construidas en las sociedades patriarcales.

Tabla 2.

Relación entre amor y felicidad en las mujeres.

Mujeres que aceptan el mito de la felicidad

"Yo sí me sentiría incompleta [sin una relación de pareja]. 0 sea, ahora mismo no tengo pareja y estoy súper bien, creo que son etapas. En plan, ¿toda una vida sin pareja? Es que no, yo no me veo". (G2, mujer 4)

"Yo alguna vez me he llegado a sentir incompleta por no tener pareja, o no pareja como tal, pero alguien similar a una pareja. Necesitaba que alguien estuviese ahí y me dijese cosas buenas de mí misma". (G4, mujer 22)

"Ahora mi novio me dice que se va de Erasmus y me muero, me muero". (G2, mujer 1)

"Yo por ejemplo ahora no tengo pareja y yo me considero una persona feliz porque tengo mis momentos felices. Pero, claro, es lo que me acaba de decir [mujer 17], yo no sé si en otro momento de mi vida va a ser igual". (G3, mujer 15)
Mujeres que rechazan el mito de la felicidad

"Yo, mi felicidad la pongo en mí. 0 sea, no, nadie depende de mí. Nadie tiene mi felicidad. (...) Mi felicidad depende de mí y sola estoy muy bien. Y yo quiero, yo quiero estar contigo, pero sin ti puedo estar". (G2, mujer 7)

"Es muy importante la independencia, o por lo menos para mí en la pareja. Dejar de ser yo misma no es una opción y no es negociable. 0 sea, yo puedo llegar a acuerdos y puedo comprometerme a ciertas cosas, igual que mi pareja, pero no quiero dejar de ser quien soy". (G2, mujer 8)

"Yo creo que una pareja tiene que ser como un complemento, no el centro de tu vida, porque tienes que ser la protagonista de tu vida y la persona tiene que ser un complemento que te apoye, no que, por tener pareja, cambie tu vida, tus objetivos 0 tus ideas". (G4, mujer 21) 


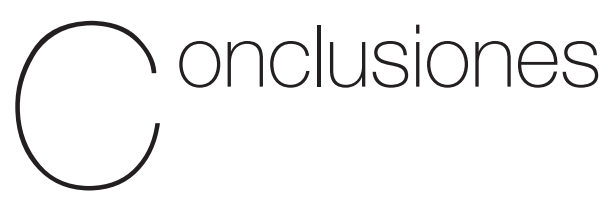

El logro de la igualdad de género y del empoderamiento de las mujeres es uno los objetivos prioritarios a nivel mundial en la actualidad (Organización de las Naciones Unidas, 2015). No obstante, aunque se ha avanzado mucho en las últimas décadas, todavía se está lejos de alcanzar la igualdad real. En las sociedades formalmente igualitarias, la desigualdad de género continúa perpetuándose a través de distintos mecanismos. La significación del amor, tomándolo como una construcción social y cultural cargada de simbología, es un mecanismo simbólico de producción y reproducción del poder patriarcal. Los resultados del estudio reportan que los diferentes significados que hombres y mujeres han construido en torno al amor generan las condiciones simbólicas que mantienen la asimetría y la jerarquización en las relaciones íntimas heterosexuales. En este sentido, la aproximación a las creencias sobre el amor ha permitido comprender el papel que pueden tener en la reproducción de la desigualdad, en una muestra de jóvenes de la Comunidad de Madrid.

Cabe mencionar que el estudio cuenta con algunas limitaciones. La muestra no tiene la misma proporción de mujeres que de hombres. Esto se debe a que las personas que participaron en la investigación son estudiantes de Magisterio en Educación Primaria, una profesión feminizada debido a la división sexual del trabajo y de los roles sociales (Sánchez, 2012). Los grupos focales pueden dar lugar a cierta deseabilidad social por la presión del grupo; no obstante, los grupos fueron no mixtos y se desarrollaron en un ambiente conocido para evitar cualquier tipo de sesgo. En futuras investigaciones sería apropiado analizar otras variables -como la edad, la tenencia de pareja, la religiosidad, el nivel educativo- que pudieran estar relacionadas con las creencias sobre el amor y sobre las relaciones íntimas. Por último, partiendo del amor como construcción que depende del contexto sociohistórico, habría que explorar las implicaciones de estas creencias en contextos culturales diversos.

En conclusión, a pesar de las limitaciones, los resultados pueden ayudar a poner en marcha acciones preventivas que aborden el amor desde un enfoque integral, poniendo el foco en la socialización diferencial y en las desigualdades de género (Ferrer \& Bosch, 2013). La transformación de las relaciones históricamente desiguales entre hombres y mujeres requiere de una actualización de las acciones educativas (Colás, 2007), introduciendo la coeducación y la educación afectivo-sexual en el currículo educativo (Bonilla \& Rivas, 2018; Venegas, 2010). El ámbito de la educación formal es un lugar privilegiado para abordar el amor, los mitos románticos, las dinámicas relacionales y los estereotipos de género (Cerreti \& Nararro, 2018; Rodríguez et al., 2015). Las relaciones íntimas no pueden construirse sobre la necesidad, la dependencia y la desigualdad; hay que resignificar el amor, proponer alternativas que rompan con los mandatos patriarcales y generar las condiciones simbólicas que posibiliten la igualdad, la independencia y la no violencia en las relaciones íntimas. 


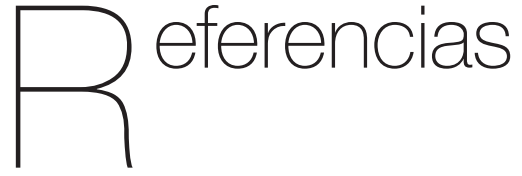

Baron, R. A. \& Byrne, D. (2005). Psicología social. España: Pearson Educación.

Alfama, E., Cruells, M. \& De La Fuente, M. (2014). Medir la igualdad de género: Debates y reflexiones a partir de una propuesta de sistema de indicadores clave. Athenea Digital, 14(4), 209-235. https://doi. org/10.5565/rev/athenea.1460.

Barrón, A., Martínez-Iñigo, D., de Paúl, P. \& Yela, C. (1999). Romantic beliefs and myths in Spain. The Spanish Journal of Psychology, 2, 64-73. https://doi. org/10.1017/S1138741600005461.

Bohórquez, C., \& Rodríguez-Cárdenas, D. (2014). Percepción de Amistad en Adolescentes: el Papel de las Redes Sociales. Revista Colombiana de Psicología, 23(2), 325-338. https://doi. org/10.15446/rcp.v23n2.37359.

Bonilla, E., \& Rivas, E. (2018). Propiedades psicométricas de la versión reducida de la Escala de Mitos sobre el Amor en una muestra de estudiantes colombianos. Suma Psicológica, 25(2), 162-170. http:// dx.doi.org/10.14349/sumapsi.2018.v25. n2.8.

Bosch, E., Ferrer, V. A. \& Alzamora, A. (2005). Algunas claves para una psicoterapia de orientación feminista en mujeres que han padecido violencia de género. Feminismo/s, 6, 121-136.

Bosch, E., Ferrer, V. A., Ferreiro, V., \& Navarro, C. (2013). La violencia contra las mujeres: el amor como coartada. Madrid: Anthropos Editorial.

Cassepp-Borges, V., \& Leôni, M. (2009). Versión reducida de la Escala Triangular del Amor: características del sentimiento en Brasil. Interamerican Journal of Psychology, 43(1), 30-38.

Cerreti, G. \& Nararro, C. (2018). Myths of romantic love: gender perspectives in adolescents dating. AGAbout Gender, 7(13), 76-95. https://doi.org/10.15167/2279-5057/ AG2018.7.13.480.

Chaves, A. R. (2012). Masculinidad y feminidad: ¿De qué estamos hablando? Revista Electrónica Educare, 16, 5-13. https://doi. org/10.15359/ree.16-Esp.1.

Colás, P. (2007). La construcción de la identidad de género: enfoques teóricos para fundamentar la investigación e intervención educativa. Revista de Investigación Educativa, 25(1), 151-166.

Cubells, J. \& Calsamiglia, A. (2015). El repertorio del amor romántico y las condiciones de posibilidad para la violencia machista. Universitas Psychologica, 14(5), 16811694. http://dx.doi.org/10.11144/Javeriana. upsy14-5.rarc.

De Beauvoir, S. (2008). El segundo sexo. Madrid: Ediciones Cátedra.

Espinar, E. (2007). Las raíces socioculturales de la violencia de género. Escuela Abierta, 10, 23-48.

Esteban, M. L. (2009). Identidades de género, feminismo, sexualidad y amor: los cuerpos como agentes. Política y Sociedad, 46(1-2), 27-41. 
Esteban, M. L. \& Távora, A. (2008). El amor romántico y la subordinación social de las mujeres: revisiones y propuestas. Anuario de Psicología, 39(1), 59-73.

Expósito, F., Moya, M. C., \& Glick, P. (1998). Sexismo ambivalente: medición y correlatos. Revista de Psicología Social, 13(2), 159-169. http://doi. org/10.1174/021347498760350641

Ferreiro, V., Caro, C., Ferrer, V.A., Casado, I. \& Casado, R.M. (2018). Creencias sobre el 'amor' en la pareja: Estudio cualitativo en alumnado universitario de diferentes contextos geográficos. En Investigación y género: Reflexiones desde la investigación para avanzar en igualdad (pp. 216-228). Sevilla: SIEMUS.

Ferrer, V. \& Bosch, E. (2013). Del amor romántico a la violencia de género: Para una coeducación emocional en la agenda educativa. Profesorado: Revista de currículum y formación del profesorado, 17(1), 107-122.

García, E. (2006). El espejismo de la igualdad: el peso de las mujeres y de lo femenino en las iniciativas de cambio institucional. Otras Miradas, 6(1), 24-30.

García, A. \& Cedillo, P. (2011). Tras los pasos del amor: un recuento desde las Ciencias Sociales. Estudios Sociológicos, 29, 551-602.

García, M. E., Garnica, M. C., González, N., Márquez, M., Martín, M., Pérez, M. M. T., \& Vico, M. N. (2011). Las mujeres viven la relación romántica diferente al hombre. Reidocrea, 1, 95-100.

Glaser, B. G., \& Strauss, A. L. (1999). Discovery of Grounded Theory. New York: Routledge.
Glick, P., \& Fiske, S. T. (1996). The Ambivalent Sexism Inventory: Differentiating Hostile and Benevolent Sexism. Journal of Personality and Social Psychology, 70(3), 491-512. http://doi.org/10.1037/0022-3514.70.3.491

Hamui-Sutton, A. \& Varela-Ruiz, M. (2013). La técnica de grupos focales. Investigación en Educación Médica, 2(5), 55-60.

Hernández, R., Fernández, C., \& Baptista, M. P. (2010). Metodología de la Investigación. México: McGraw-Hill.

Herrera, C. (2014). Amor romántico y desigualdad de género. Revista Feminista Casa de la Mujer, 20(2), 79-95.

Krueger, R. A. (1991). El grupo de discusión: guía práctica para la investigación aplicada. España: Pirámide.

Lagarde, M. (2001). Claves feministas para la negociación del amor. Managua: Puntos de encuentro.

Leal, A. (2007). Nuevos tiempos, viejas preguntas sobre el amor: Un estudio con adolescentes. Posgrado y Sociedad, 7(2), 50-70.

Organización de las Naciones Unidas (2015). Transformar nuestro mundo: la Agenda 2030 para el Desarrollo Sostenible. Extraído de https://www.un.org/ga/search/view_ doc.asp?symbol=A/RES/70/1\&Lang=S

Pascual, A. (2016). Sobre el mito del amor romántico: amores cinematográficos y educación. DEDICA, Revista de Educação e Humanidades, 10, 63-78.

Rodríguez, Y., Lameiras, M. \& Carrera, M. V. (2015). Amor y sexismo: una peligrosa relación en los y las adolescentes gallegos/ 
as. Revista de Estudios e Investigación en Psicologíay Educación, Extr. (2), 11-14. http:// doi.org/10.17979/reipe.2015.0.02.234.

Rodríguez-Santero, J., García-Carpintero, M. A., \& Porcel, A. M. (2017). Los estilos de amor en estudiantes universitarios: diferencias en función del sexo-género. Revista Internacional de Sociología, 75(3), e073. http://dx.doi.org/10.3989/ ris.2017.75.3.15.171.

Sánchez, A. (2012). La división sexual del trabajo docente: procesos discursivos y realidades sobrevenidas. Revista Electrónica Interuniversitaria de Formación del Profesorado, 15(3), 85-91.

Venegas, M. (2010). Educar las relaciones afectivosexuales, prevenir las diferentes formas de violencia de género. Trabajo Social Global, 1(2), 162-182.

Verdú, A. D. (2014). Igualdad y desigualdad de género en los imaginarios sociales del amor de pareja heterosexual. Cuestiones de género: de la igualdad y la diferencia, (9), 402-427. 